DPNU-96-63

December 1996

\title{
Effective Messenger Sector from Dynamical Supersymmetry Breaking
}

\author{
Naoyuki Haba, Nobuhito Maru and Takeo Matsuoka \\ Department of Physics \\ Nagoya University \\ Nagoya 464-01, Japan \\ haba@eken.phys . nagoya-u.ac.jp \\ maru@eken.phys.nagoya-u.ac.jp \\ matsuoka@eken.phys.nagoya-u.ac.jp
}

\begin{abstract}
In the framework of the dynamical supersymmetry breaking we construct the messenger sector as the effective theory of supersymmetry breaking sector, which is based on $S U(3) \times S U(2)$ model of Affleck, Dine and Seiberg. In our model, messenger superfields with the non-renormalizable interaction are contained. By minimizing the scalar potential, we show that the supersymmetry breaking can be communicated to the visible sector without breaking QCD color. In this model there appear various scales. Supersymmetry breaking scale turns out to be the intermediate scale $\left(\sim 10^{10} \mathrm{GeV}\right)$ between the GUT scale and the soft supersymmetry breaking scale.
\end{abstract}




\section{Introduction}

In order for supersymmetry to be relevant to the real world, it must be broken. Dynamical supersymmetry breaking [1] is one of the attractive scenarios because it can solve the gauge hierarchy problem elegantly by the dimensional transmutation. From the phenomenological point of view, it is a problem of how supersymmetry breaking is communicated to the visible sector, in other words, what is the messenger interaction. At present, we know of two scenarios in this regard. One is a usual hidden sector scenario [2], in which the gravity plays the role of the messenger of supersymmetry breaking. Another is a gauge-mediated supersymmetry breaking scenario [3, 4], in which the gauge interactions play the role of the messenger of supersymmetry breaking. One of the attractive advantages of the latter scenario is that the flavor changing neutral currents (FCNC) are naturally suppressed, because gauginos, squarks, sleptons masses appear radiatively at the low energy, contrary to the hidden sector scenario and are proportional to the flavor-blind gauge coupling constants squared. Furthermore, this scenario is highly predictive because the superparticle spectrum is calculable.

Recently, Dasgupta, Dobrescu and Randall [5] showed that the true vacuum does not preserve QCD color in the minimal gauge-mediated supersymmetry breaking scenario. In the minimal gauge-mediated supersymmetry breaking [4], the messenger superpotential is of the form

$$
W_{\text {mes }}=k_{1} \phi^{+} \phi^{-} X+\frac{1}{3} \lambda X^{3}+k_{3} X l \bar{l}+k_{4} X q \bar{q},
$$

where $q, \bar{q}$ are messenger quarks in $\mathbf{3}$ and $\overline{\mathbf{3}}$ of the color $S U(3)_{C}, l, \bar{l}$ are messenger leptons in 2 of $S U(2)_{L}, X$ is a singlet and $\phi^{+}, \phi^{-}$have $+1,-1$ charges of the messenger group $U(1)_{m}$. In this conventional model the $\phi^{+} \phi^{-} X$ term communicates supersymmetry breaking from the charged fields under $U(1)_{m}$ to the singlet $X$ and the $X q \bar{q}$ and $X l \bar{l}$ terms link the singlet with the messenger quarks and leptons. From Eq. (1.1) the 
scalar potential of the messenger sector is given by

$$
\begin{aligned}
V_{\text {mes }}= & k_{1}^{2}|X|^{2}\left(\left|\phi^{+}\right|^{2}+\left|\phi^{-}\right|^{2}\right)+\left|k_{1} \phi^{+} \phi^{-}+\lambda X^{2}+k_{3} l \bar{l}+k_{4} q \bar{q}\right|^{2} \\
& +k_{3}^{2}|X|^{2}\left(|l|^{2}+|\bar{l}|^{2}\right)+k_{4}^{2}|X|^{2}\left(|q|^{2}+|\bar{q}|^{2}\right) \\
& +M^{\prime 2}\left(\left|\phi^{+}\right|^{2}+\left|\phi^{-}\right|^{2}\right)+\frac{g_{1}^{2}}{2}\left(\left|\phi^{+}\right|^{2}-\left|\phi^{-}\right|^{2}\right)^{2}
\end{aligned}
$$

where the second last term represents the soft supersymmetry breaking term with the mass $M^{\prime 2}<0$ and the last term is $U(1)_{m}$ D-term. Since the soft supersymmetry breaking mass is negative, the vacuum expectation values (VEVs) of $\phi^{+}, \phi^{-}$become non-zero. Then the supersymmetry breaking is transmitted to the singlet through the first term in Eq. (1.1). Through the minimization of Eq. (1.2), $X$ and $F_{X}$ are expected to develop non-zero VEVs. If this is the case, the supersymmetry breaking is transmitted to the visible sector radiatively by way of the last two terms in Eq. (1.1). As pointed out in Ref. [5], however, careful study shows that QCD color is violated in the true vacuum. We are now at the stage that we have to reconsider the gaugemediated supersymmetry breaking scenario and extend the messenger sector and/or the messenger interactions. Many attempts [6] have been made on the variations of the minimal gauge-mediated supersymmetry breaking.

In this paper we construct the messenger sector as the effective theory of supersymmetry breaking sector, which is based on $S U(3) \times S U(2)$ model of Affleck, Dine, and Seiberg [7]. In the conventional model of the gauge-mediated supersymmetry breaking all of the messenger matter fields and interactions are put by hand. The messenger superpotential (1.1) does not incorporate the F-type of supersymmetry breaking. The supersymmetry breaking is put into the messenger sector only through the soft scalar masses of $\phi^{+}$and $\phi^{-}$. In contrast with the conventional model, our messenger sector superpotential is the effective superpotential of the supersymmetry breaking sector and no superfields are added to the messenger sector superpotential. This messenger superpotential yields the F-type of supersymmetry breaking. What we would like to emphasize here is that it does not seem to be appropriate to analyze the supersym- 
metry breaking sector and the messenger sector separately. From the beginning the messenger superfields with the non-renormalizable interaction are contained in the supersymmetry breaking sector. Furthermore, we show that supersymmetry breaking can be communicated to the visible sector without breaking QCD color.

As discussed in Ref. [4], gaugino masses are generated at one-loop, whereas squark and slepton masses are generated at two-loop. As a result, FCNC are naturally suppressed. We also take the effects of gravitational interaction into account in calculating masses of gauginos, squarks and sleptons. It is found that these effects are not important compared with gauge interaction effects. In the present framework, we find that supersymmetry is broken at the intermediate scale $\left(\sim 10^{10} \mathrm{GeV}\right)$ between the GUT scale and the soft supersymmetry breaking scale. The masses of the messenger fields $\left(10^{6 \sim 9} \mathrm{GeV}\right)$ are in the intermediate scale between supersymmetry breaking scale and soft supersymmetry breaking scale.

This paper is organized as follows. In Section 2, the dynamical supersymmetry breaking sector is discussed. We construct the messenger sector as the effective theory of dynamical supersymmetry breaking sector and minimize the scalar potential. In Section 3, we estimate various scales in our model. Summary is found in Section 4.

\section{Messenger sector as the effective theory of super- symmetry breaking sector}

Our model is based on the $S U(3) \times S U(2)$ model [7], which breaks supersymmetry dynamically. This model has the $S U(3) \times S U(2)$ gauge group, a global $U(1)_{m}$ and a non-anomalous global R-symmetry. In our model this $U(1)_{m}$ is gauged from the beginning. The representations and charges of the matter fields are summarized in Table 1 . Note that the singlet superfield $\bar{E}$ is included to cancel $U(1)_{m}$ anomaly and

that vector-like superfields $f \equiv(q, l), \bar{f} \equiv(\bar{q}, \bar{l})$ are involved. Here we introduce the parameter $r$ which specifies the $U(1)_{R^{-c h a r g e s}}$ for each superfields and we assume 
that the sum of R-charge of $f$ and $\bar{f}$ is 0 . Under the standard model gauge group $G_{\text {standard }} \equiv S U(3)_{C} \times S U(2)_{L} \times U(1)_{Y}, Q, \bar{U}, \bar{D}, L$ and $\bar{E}$ are neutral and $q, \bar{q}, l$ and $\bar{l}$ are transformed as

$$
q:(\mathbf{3}, \mathbf{1},-2 / 3), \quad \bar{q}:(\overline{\mathbf{3}}, \mathbf{1}, 2 / 3), \quad l:(\mathbf{1}, \mathbf{2}, 1), \quad \bar{l}:(\mathbf{1}, \mathbf{2},-1),
$$

respectively.

\section{Table 1}

The tree level superpotential consistent with the symmetries is

$$
W_{\text {tree }}=\lambda_{1} Q \bar{D} L+\frac{\kappa^{\prime}}{M^{2}}(Q \bar{D} L) f \bar{f}+\frac{\lambda_{2}^{\prime}}{M^{5}}(Q \bar{U} L) \bar{E}(\operatorname{det} Q \bar{Q}),
$$

where $\bar{Q}=(\bar{U}, \bar{D})$. In Eq. (2.2) the first term is a renormalizable term which has been treated in Ref. [7]. The second and the third terms are non-renormalizable terms which will be at most cubic terms of the $S U(3) \times S U(2)$ gauge invariant operatorst. The coupling constants $\lambda_{2}^{\prime}$ and $\kappa^{\prime}$ are taken to be of order $O(1) . \lambda_{1}$ is assumed to be very small so that the theory becomes weakly coupled.

To analyze the model we first consider the case in which the superpotential is absent. Under the condition that $g_{3} \gg g_{2} \gg g_{1}$, where $g_{3}, g_{2}$ and $g_{1}$ represent $S U(3), S U(2)$ and $U(1)_{m}$ gauge coupling constants, respectively, there exist the D-flat directions for $S U(3)$ and $S U(2)[7$

$$
\langle Q\rangle=\left(\begin{array}{ll}
a & 0 \\
0 & b \\
0 & 0
\end{array}\right),\langle\bar{U}\rangle=\left(\begin{array}{l}
a \\
0 \\
0
\end{array}\right),\langle\bar{D}\rangle=\left(\begin{array}{l}
0 \\
b \\
0
\end{array}\right),\langle L\rangle=\left(0, \sqrt{a^{2}-b^{2}}\right) .
$$

Here $a$ and $b$ are taken as real and positive parameters with $a \geq b$. Gauge symmetries are completely broken along these flat directions and the one-instanton effect induces

\footnotetext{
${ }^{1}$ Other non-renormalizable terms are highly suppressed by the scale $M$, which will be set to be the Planck scale later.
} 
the non-perturbative superpotential [7]

$$
W_{\mathrm{dyn}}=\frac{\Lambda_{3}^{7}}{\operatorname{det} Q \bar{Q}},
$$

where $\Lambda_{3}$ is the scale where $S U(3)$ gauge coupling blows up. Note that we consider the case $\Lambda_{3} \gg \Lambda_{2}$, where $\Lambda_{2}$ is the scale where $S U(2)$ gauge coupling diverges 2 .

If we turn on the tree level superpotential, the flat directions are lifted. In our case VEVs of the fields are close to those of Ref. [7], $v \sim \Lambda_{3} / \lambda_{1}^{1 / 7} \gg \Lambda_{3}$. The vacuum energy is $V \sim \lambda_{1}^{2} v^{4}>0$ and then supersymmetry is broken. The moduli space is described in terms of the $S U(3) \times S U(2)$ gauge invariant operators [9] listed in Table 2. From Eqs. (2.2) and (2.4) below the scale $\Lambda_{3}$ we have the effective superpotential

$$
\begin{aligned}
W_{\mathrm{eff}} & =\lambda_{1} X_{1}+\frac{\Lambda_{3}^{7}}{X_{3}}+\frac{\kappa^{\prime}}{M^{2}} X_{1} f \bar{f}+\frac{\lambda_{2}^{\prime}}{M^{5}} X_{2} \bar{E} X_{3}, \\
& =\lambda_{1} v^{2} Y+\frac{\lambda_{1} v^{4}}{X}+\kappa Y f \bar{f}+\lambda_{2} P N X,
\end{aligned}
$$

where in the second equality we rescale the gauge invariant operators as $X_{1}=v^{2} Y, X_{2}=$ $v^{2} N, X_{3}=v^{3} X$ and $\bar{E}=P$. Here we introduce the notations as $\kappa \equiv \kappa^{\prime}(v / M)^{2}$ and $\lambda_{2} \equiv \lambda_{2}^{\prime}(v / M)^{5}$. Equation (2.6) represents the effective theory of the supersymmetry breaking sector. Since $N$ and $P$ have $U(1)_{m}$ charges -1 and +1 , respectively, these superfields correspond to $\phi^{-}$and $\phi^{+}$in the minimal model [4]. Contrary to Ref. [四] we add no superfields to the effective superpotential. In the present model we have $\left\langle F_{Y}\right\rangle \sim \lambda_{1} v^{2}$. Then, the supersymmetry breaking is communicated to the messenger fields $f$ and $\bar{f}$ through $\kappa Y f \bar{f}$ term. It is worth noting that the $U(1)_{m}$ gauge interaction and $\lambda_{2} P N X$ term do not play an essential role in communicating the supersymmetry breaking to the messenger fields.

\section{Table 2}

\footnotetext{
${ }^{2}$ If we consider the case $\Lambda_{2} \gg \Lambda_{3}$, supersymmetry is broken due to the quantum deformation of the moduli space [8].
} 
In order to analyze the scalar potential in our model, it is necessary to calculate the effective Kähler potential. Under the condition $\lambda_{1} \ll 1$, we can calculate the effective Kähler potential using the procedure given by Poppitz and Randall [10 because the theory is weakly coupled and the gauge symmetries are completely broken. The effective Kähler potential is given by

$$
K=3\left(t+\frac{B}{t}\right),
$$

where

$$
\begin{aligned}
t & \equiv\left(A+\sqrt{A^{2}-B^{3}}\right)^{1 / 3}+\left(A-\sqrt{A^{2}-B^{3}}\right)^{1 / 3} \\
A & \equiv \frac{1}{2}\left(X_{1}^{\dagger} X_{1}+X_{2}^{\dagger} X_{2}\right)=\frac{1}{2} v^{4}\left(Y^{\dagger} Y+N^{\dagger} N\right), \\
B & \equiv \frac{1}{3}\left(X_{3}^{\dagger} X_{3}\right)^{1 / 2}=\frac{1}{3} v^{3}\left(X^{\dagger} X\right)^{1 / 2} .
\end{aligned}
$$

The inverse of the effective Kähler metric is

$$
K^{j^{*} i}=\left(\begin{array}{ccc}
\left(\frac{t}{v^{2}}\right)^{2}+\frac{2}{t} Y^{\dagger} Y & \frac{2}{t} Y^{\dagger} N & \frac{2}{t} Y^{\dagger} X \\
\frac{2}{t} N^{\dagger} Y & \left(\frac{t}{v^{2}}\right)^{2}+\frac{2}{t} N^{\dagger} N & \frac{2}{t} N^{\dagger} X \\
\frac{2}{t} X^{\dagger} Y & \frac{2}{t} X^{\dagger} N & \frac{2 t}{v^{3}}|X|+\frac{2}{t} X^{\dagger} X
\end{array}\right)
$$

for $i, j=Y, N$ and $X$. Since $P(=\bar{E}), f$ and $\bar{f}$ have no $S U(3)$ charge, their components of the effective Kähler potential are assumed to be of canonical form. From Eqs. (2.6) and (2.9) the scalar potential of the effective theory is given by

$$
\begin{aligned}
V= & W_{j^{*}} K^{j^{*} i} W_{i}+\frac{g_{1}^{2}}{2}\left(|P|^{2}-|N|^{2}\right)^{2}+\left(M_{P}^{2}|P|^{2}+M_{N}^{2}|N|^{2}\right), \\
= & \frac{2}{t}\left|\lambda_{1} v^{2} Y+\kappa Y f \bar{f}+2 \lambda_{2} P N X-\frac{\lambda_{1} v^{4}}{X}\right|^{2} \\
& +\left(\frac{t}{v^{2}}\right)^{2}\left(\lambda_{2}^{2}|X|^{2}|P|^{2}+\left|\lambda_{1} v^{2}+\kappa f \bar{f}\right|^{2}\right)+\frac{2 t}{v^{3}}|X|\left|\lambda_{2} P N-\frac{\lambda_{1} v^{4}}{X^{2}}\right|^{2} \\
& +\lambda_{2}^{2}|N|^{2}|X|^{2}+\kappa^{2}|Y|^{2}\left(|f|^{2}+|\bar{f}|^{2}\right) \\
& +\frac{g_{1}^{2}}{2}\left(|P|^{2}-|N|^{2}\right)^{2}+\left(M_{P}^{2}|P|^{2}+M_{N}^{2}|N|^{2}\right),
\end{aligned}
$$

where the second last term is $U(1)_{m}$ D-term, because $U(1)_{m}$ is gauged and $U(1)_{m}$ D-flatness condition is not imposed. The last term represents two-loop generated soft 
supersymmetry breaking mass term. Note that $M_{P}^{2}$ and $M_{N}^{2}$ are negative and of order $O\left(\left(\frac{g_{1}^{2}}{16 \pi^{2}}\right)^{2} \lambda_{1}^{2} v^{2}\right)$.

By minimizing the scalar potential (2.10) under the conditions

$$
\kappa \gg \lambda_{1}, \quad \frac{g_{1}^{2}}{16 \pi^{2}} \lambda_{1} \gg \lambda_{2},
$$

we obtain VEVs which are in the vicinity of the $S U(3), S U(2)$ D-flat direction [7], namely

$$
\begin{aligned}
& X=v_{X}+x, \quad|x| \ll v_{X}, \\
& Y=v_{Y}+y, \quad|y| \ll v_{Y}, \\
& |f|,|\bar{f}|,|P| \text { and }|N| \ll v,
\end{aligned}
$$

where $v_{X} \equiv a^{2} b^{2} / v^{3}, v_{Y} \equiv a^{2} \sqrt{a^{2}-b^{2}} / v^{2}$ and $x, y$ represent the fluctuation around $v_{X}, v_{Y}$, respectively. In the minimization it is important that the effective Kähler potential has of the non-canonical form. We can easily derive

$$
\langle f\rangle=\langle\bar{f}\rangle=0
$$

from the stationary conditions for $f$ and $\bar{f}$. This shows that QCD color is not broken. By calculating the minimization conditions with respect to $N$ and $P$ we obtain

$$
\begin{aligned}
& |\langle P\rangle| \simeq \frac{1}{g_{1}} \sqrt{-M_{P}^{2}} \sim \frac{g_{1}}{16 \pi^{2}} \lambda_{1} v, \\
& |\langle N\rangle| \simeq \frac{\lambda_{2}}{\lambda_{1}}|\langle P\rangle| \sim \frac{g_{1}}{16 \pi^{2}} \lambda_{2} v
\end{aligned}
$$

The order of VEVs of the fluctuation $x$ and $y$ is

$$
|\langle x\rangle| \sim|\langle y\rangle| \sim O\left(\left(\frac{g_{1}^{2}}{16 \pi^{2}}\right)^{2} \lambda_{2}^{2} v\right) \ll v_{X}, v_{Y} .
$$

As a consequence, our analysis is found to be self-consistent. 


\section{Visible sector and Estimation of the scales}

Supersymmetry breaking in the messenger sector is transmitted to gauginos, squarks and sleptons in the visible sector radiatively through the interaction $\kappa Y f \bar{f}$ in Eq.(2.6). At one-loop we can obtain the masses for $S U(3)_{C}, S U(2)_{L}$ and $U(1)_{Y}$ gauginos

$$
m_{\lambda_{i}} \sim \frac{g_{i}^{2}}{16 \pi^{2}} \frac{\left\langle F_{Y}\right\rangle}{\langle Y\rangle} \sim \frac{g_{i}^{2}}{16 \pi^{2}} \lambda_{1} v
$$

where $g_{i}$ stands for the corresponding gauge coupling of the standard model. Taking $m_{\lambda_{i}}=10^{2.0 \pm 0.5} \mathrm{GeV}$, we obtain

$$
\lambda_{1}\left(\frac{v}{M_{\text {Planck }}}\right) \sim 10^{-13.3 \pm 0.5}
$$

where $\frac{g_{i}^{2}}{16 \pi^{2}} \simeq 10^{-2.5}$ is used. On the other hand, the soft supersymmetry breaking masses for squarks and sleptons are induced at two-loop. They are given by

$$
m_{\phi_{i}}^{2} \sim\left(\frac{g_{i}^{2}}{16 \pi^{2}} \frac{\left\langle F_{Y}\right\rangle}{\langle Y\rangle}\right)^{2} \sim\left(\frac{g_{i}^{2}}{16 \pi^{2}} \lambda_{1} v\right)^{2}
$$

Here we focus on the estimation of the various scales. In the supersymmetry breaking sector we have a scale $M$ which supresses the non-renormalizable interactions in Eq.(2.2). It is natural to take this scale $M$ as $M_{\text {Planck }}$. This implies that we have to take the effects of the gravitational interaction into account in calculating the masses for the gauginos, the squarks, and the sleptons. The scalar mass terms which are induced by gravity come from the D-term

$$
\int d^{4} \theta\left(\frac{Q^{\dagger} Q}{M_{\text {Planck }}^{2}}+\cdots\right) \Phi_{i}^{\dagger} \Phi_{i},
$$

where $\Phi_{i}$ are superfields of the standard model and $i$ denotes flavor index. Therefore, the gravity-induced scalar masses are

$$
\begin{aligned}
m_{\phi_{i}}(\text { grav }) & \sim \frac{\langle F\rangle}{M_{\text {Planck }}}, \\
& \sim \lambda_{1}\left(\frac{v}{M_{\text {Planck }}}\right)^{2} M_{\text {Planck }} \ll 10^{2.5 \pm 0.5} \mathrm{GeV}
\end{aligned}
$$


where $\langle F\rangle$ is the F-term in the supersymmetry breaking sector. The last inequality implies that we consider only the case in which the gravitational effects is negligible. From this inequality we obtain

$$
\lambda_{1}\left(\frac{v}{M_{\text {Planck }}}\right)^{2} \ll 10^{-15.8 \pm 0.5}
$$

where $M_{\text {Planck }} \simeq 10^{18.3} \mathrm{GeV}$ is used.

On the other hand, gaugino mass terms which are induced by gravity arise via the term

$$
\int d^{2} \theta \frac{X_{1}}{M_{\text {Planck }}^{3}} W^{\alpha} W_{\alpha}=\int d^{2} \theta\left(\frac{v}{M_{\text {Planck }}}\right)^{2} \frac{Y}{M_{\text {Planck }}} W^{\alpha} W_{\alpha},
$$

where $W^{\alpha}$ is a field strength superfield. Thus, the gravity-induced gaugino massess become

$$
m_{\lambda_{i}}(\text { grav }) \sim \lambda_{1}\left(\frac{v}{M_{\text {Planck }}}\right)^{4} M_{\text {Planck }} .
$$

From Eq. (3.2) and the inequality (3.7) we find that

$$
m_{\lambda_{i}} \gg m_{\lambda_{i}}(\operatorname{grav})
$$

Namely, the gauge-mediated contribution to the gaugino mass is dominant compared with the gravity-mediated contribution.

Taking the condition $\lambda_{1} \ll \kappa \sim\left(\frac{v}{M_{\text {Planck }}}\right)^{2}$ into account together with Eqs. and (3.7), we obtain the allowed range of parameters

$$
\begin{aligned}
10^{-4.4 \pm 0.2} & \ll \frac{v}{M_{\text {Planck }}} \ll 10^{-2.5} \\
10^{-10.8 \pm 0.5} & \ll \lambda_{1} \ll 10^{-8.9 \pm 0.3} .
\end{aligned}
$$

If we take $\lambda_{1} \sim 10^{-9.3}$ as an example, various scales in the model are determined as,

$$
\begin{aligned}
v & \sim 10^{14.3} \mathrm{GeV} \\
\Lambda & \sim 10^{13.0} \mathrm{GeV} \\
\sqrt{F} & \sim \sqrt{\lambda_{1} v^{2}} \sim 10^{9.6} \mathrm{GeV} \\
m & \sim \kappa v \sim 10^{6.3} \mathrm{GeV} \\
m_{\lambda_{i}} & \sim 10^{2.5} \mathrm{GeV}
\end{aligned}
$$


where $m$ means the mass of $f$ and $\bar{f}$. Equation (3.5) also represents the order of the gravitino mass. In the present example the gravitino mass is $\sim 10^{1.5} \mathrm{GeV}$. We note that supersymmetry breaking scale $\sqrt{F}$ turns out to be the intermediate scale between the GUT scale and the soft supersymmetry breaking scale.

\section{Summary}

We showed that the messenger sector can be considered as the effective theory of supersymmetry breaking sector. No matter superfields and interactions are added in the messenger sector. In other words, all interactions in our messenger sector are derived from the supersymmetry breaking sector. Using this effective theory we also showed that supersymmetry breaking can be communicated to the visible sector without breaking QCD color.

In the present framework, the essential role of commuinicating the supersymmetry breaking to the visible sector is played by the $\kappa Y f \bar{f}$ term in the effective superpotential. We do not need to rely on the $U(1)_{m}$ gauge interaction and also on the $P N X$ term in the superpotential. This situation is in sharp contrast to that in Ref. [4]. In fact, when we do not introduce the field $P(=\bar{E})$ and the $U(1)_{m}$ gauge symmetry, we can make the model simpler. Even in that case the main point of our results remains unchanged.

There appear various scales in the present model. We also estimate the gravitational effects in calculating masses for gauginos, squarks, and sleptons. It is found that gauge-mediated contributions are dominant compared with gravity-mediated contributions. As a typical example, we found that gauge symmetry breaking scale $(v)$ in supersymmetry breaking sector is $10^{14.3} \mathrm{GeV}$, the scale of $S U(3)$ dynamics $\left(\Lambda_{3}\right)$ in $S U(3) \times S U(2)$ model is $10^{13.0} \mathrm{GeV}$, supersymmetry breaking scale $(\sqrt{F})$ is $10^{9.6} \mathrm{GeV}$ and the mass of messenger fields $(\kappa v)$ is $10^{6.3} \mathrm{GeV}$.

In the visible sector of our model, as discussed in Ref. [4], the masses for gauginos are induced at one-loop through the standard model gauge interaction. On the other 
hand, the masses for squarks, sleptons are induced at two-loop through the standard model gauge interaction. Therefore, FCNC are naturally suppressed because these masses are proportional to the square of the flavor-blind standard model gauge coupling constants.

The present model will provide a useful guide for constructing the phenomenologically viable models of the gauge-mediated supersymmetry breaking.

\section{Acknowledgements}

One of the authors, N.H. would like to thank Prof. T. Yanagida and Dr. K.-I. Izawa for useful discussions. We thank Prof. S. Kitakado for careful reading of the manuscript.

\section{References}

[1] E. Witten, Nucl. Phys. B212 (1982) 253.

[2] H.P. Nilles, Phys. Rep. 110 (1984) 1.

[3] M. Dine, W. Fischer, and M. Srednicki, Nucl. Phys. B189 (1981) 575.

S. Dimopoulos and S. Raby, Nucl. Phys. B192 (1981) 353.

M. Dine and W. Fischer, Phys. Lett. B110 (1982) 227.

M. Dine and M. Srednicki, Nucl. Phys. B202 (1982) 238.

M. Dine and W. Fischer, Nucl. Phys. B204 (1982) 346.

L. Alvarez-Gaume, M. Claudson, and M. Wise, Nucl. Phys. B207 (1982) 96.

C.R. Nappi and B.A. Orvut, Phys. Lett. B113 (1982) 175.

S. Dimopoulos and S. Raby, Nucl. Phys. B219 (1983) 479.

[4] M. Dine and A.E. Nelson, Phys. Rev. D48 (1993) 1277, hep-ph/9303230.

M. Dine, A.E. Nelson, and Y. Shirman, Phys. Rev. D51 (1995) 1362, hepph/9408384. 
M. Dine, A.E. Nelson, Y. Nir, and Y. Shirman, Phys. Rev. D53 (1996) 2658, hep-ph/9507378.

[5] I. Dasgupta, B.A. Dobrescu, and L. Randall, hep-ph/9607487.

[6] M. Dine, Y. Nir, and Y. Shirman, hep-ph/9607397.

S.P. Martin, hep-ph/9608224.

S. Dimopoulos and G.F. Giudice, hep-ph/9609344.

S. Dimopoulos, S. Thomas, and J.D. Wells, hep-ph/9609434.

[7] I. Affleck, M. Dine and N. Seiberg, Nucl. Phys. B256 (1985) 557.

[8] K. Intriligator and S. Thomas, Nucl. Phys. B473 (1996) 121, hep-th/9603158.

[9] M.A. Luty and W. Taylor, Phys. Rev. D53 (1996) 3399, hep-th/9506098.

[10] E. Poppitz and L. Randall, Phys. Lett. B336 (1994) 402, hep-th/9407185. 


\section{Figure Captions}

\section{Table 1}

The representations and charges of matter fields in supersymmetry breaking sector. The parameter $r$, which specifies $U(1)_{R}$-charges of each superfield, is left arbitrary. $r_{f}$ and $r_{\bar{f}}$ are R-charges of $f$ and $\bar{f}$, respectively.

\section{Table 2}

The gauge invariant operators which describe the moduli space. 
Table 1

\begin{tabular}{|c|c|c|c|}
\hline Particle & $S U(3) \times S U(2)$ & $U(1)_{m}$ & $U(1)_{R}$ \\
\hline$Q$ & $(3,2)$ & $1 / 6$ & $r$ \\
$\bar{U}$ & $(\overline{3}, 1)$ & $-2 / 3$ & $-4-4 r$ \\
$\bar{D}$ & $(\overline{3}, 1)$ & $1 / 3$ & $2+2 r$ \\
$L$ & $(1,2)$ & $-1 / 2$ & $-3 r$ \\
$\bar{E}$ & $(1,1)$ & 1 & $8+6 r$ \\
\hline$f$ & $(1,1)$ & 0 & $r_{f}$ \\
$\bar{f}$ & $(1,1)$ & 0 & $r_{\bar{f}}$ \\
\hline
\end{tabular}

Table 2

\begin{tabular}{|c|c|c|}
\hline & $U(1)_{m}$ & $U(1)_{R}$ \\
\hline$X_{1}=Q \bar{D} L$ & 0 & 2 \\
$X_{2}=Q \bar{U} L$ & -1 & $-4-6 r$ \\
$X_{3}=\operatorname{det} Q \bar{Q}$ & 0 & -2 \\
$\bar{E}$ & 1 & $8+6 r$ \\
\hline$f \bar{f}$ & 0 & 2 \\
\hline
\end{tabular}

